\title{
Perioral Hyperpigmentation Treated with 1,064-nm 0-switched Neodymium:Yttrium-aluminum-garnet Laser Toning
}

Wan Jin Kim

Han Kyoung $\mathrm{Cho}^{1}$

Sang Ju Lee ${ }^{2}$

${ }^{1}$ Department of Dermatology, Hanyang University Medical Center, Myongji Hospital, Goyang, Korea

${ }^{2}$ Yonsei Star Skin \& Laser Clinic, Seoul, Korea
Received February 3, 2021

Accepted March 4, 2021

\section{Correspondence}

Han Kyoung Cho

Department of Dermatology, Hanyang University Medical Center, Myongji Hospital, 55 Hwasu-ro, 14 beon-gil, Deogyang-gu, Goyang 10475, Korea Tel.: +82-31-810-7050

Fax: +82-31-810-7057

E-mail: trpchkahanmail.net

(C) Korean Society for Laser Medicine and Surgery

(c) This is an open access article distributed under the terms of the Creative Commons Attribution NonCommercial License (http://creativecommons.org/ licenses/by-nc/4.0) which permits unrestricted noncommercial use, distribution, and reproduction in any medium, provided the original work is properly cited.
The perioral region, which is one of the most conspicuous parts of the face, plays a significant role in interpersonal communication. However, the perioral region is highly susceptible to scarring and pigmentation and is difficult to treat. The causes of perioral hyperpigmentation are many, including physiologic reasons, systemic diseases, drugs, and infections. In the treatment of perioral hyperpigmentation, the cause of the disease must be diagnosed first. Management includes lifestyle modifications such as reducing the UVA exposure time, use of topical treatments including hydroquinone and retinoids, and physical therapy including laser therapy. We report a case of perioral hyperpigmentation treated with 1,064-nm Q-switched neodymium:yttrium-aluminum-garnet laser toning.

\section{Key words}

Perioral hyperpigmentation; Neodymium:yttrium-aluminum-garnet laser; Laser toning 


\section{INTRODUCTION}

The perioral region is easily exposed to many stimuli in daily life, so it is more susceptible to injury or pigmentation than other facial areas. Therefore, perioral hyperpigmentation is a disease that can be easily encountered in the clinic.'

The causes of perioral hyperpigmentation are very diverse, and the types include physiological problems such as pregnancy, genetic syndrome such as Peutz-Jeghers, endocrine disorders such as Cushing's or thyroid problem, drugs, infections and miscellaneous. ${ }^{2}$ Therefore, when diagnosing a patient with perioral hyperpigmentation, thorough physical examination on the overall skin is required. ${ }^{2,3}$

Herein, we present that the idiopathic perioral hyperpigmentation in 21-year-old woman and it was improved through 1,064-nm Q-switched neodymium (Nd):yttriumaluminum-garnet (YAG) laser toning.

\section{CASE REPORT}

A 21-year-old woman presented with complaints of hyperpigmentation around her mouth that started several years ago. As of yet, the patient has not been treated with this problem. She had no remarkable medical, family history or allergy history including atopic dermatitis. On the interview, the patient said that there was no skin change caused by the use of toothpaste or certain cosmetics. However, we did not perform any other allergy tests, including patch tests. A distinct hyperpigmented lesion bounded by vermilion border could be visually confirmed (Fig. 1).

After obtaining informed consent, we performed a total of 9 laser treatments on the perioral pigmentation area at 1-week intervals. The laser used was 1,064-nm Q-

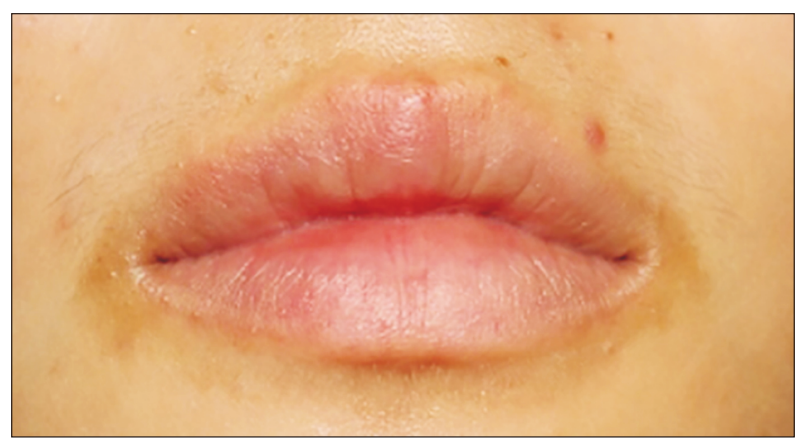

Fig. 1. Perioral hyperpigmentation clearly observed around the vermilion border. switched Nd:YAG laser (Lutronic Corp., Goyang, Korea). The parameters of the laser are as follows: $7 \mathrm{~mm}$ spot size, 1.2 to $3.0 \mathrm{~J} / \mathrm{cm}^{2}$ fluence, and $10 \mathrm{~Hz}$ repetition rate. Total shot count was 500 shots. The end point of treatment was when mild erythema appeared, and 500 shots were used because mild erythema appeared at that time. The laser was applied directly to the lip margin. Medications or ointments were not prescribed after procedure. The patient did not have any restrictions on daily life as usual. After laser treatment, perioral hyperpigmentation became much lighter and brighter (Fig. 2).

\section{DISCUSSION}

The perioral region is one of the most conspicuous parts of the face, plays a significant role in meeting and communicating with people, and gives a sense of selfsatisfaction. ${ }^{1,4}$ This region is easily exposed to many stimuli in daily life, such as eating food, brushing your teeth, or wearing a mask. Therefore, the perioral part of the face is more susceptible to injury or pigmentation than other parts of the face, and it is difficult to treat compared to other parts when it suffers from irritation or trauma. ${ }^{4}$

The causes of perioral hyperpigmentation are various, including physiologic finding, systemic diseases, drugs, and infections. ${ }^{1,2}$ Perioral hyperpigmentation treatment should first address the cause of the disease causing this, followed by life style correction, such as reducing the UVA exposure time, topical treatments including hydroquinone and retinoids, and physical therapy including laser therapy. 2,3

The Q-switched Nd:YAG laser is a technique commonly performed in the treatment of benign pigmented lesions with various wavelengths. In particular, 1,064-nm Qswitched Nd:YAG laser is often used to remove pigmented lesions such as café-au-lait macules, Becker nevi, nevus

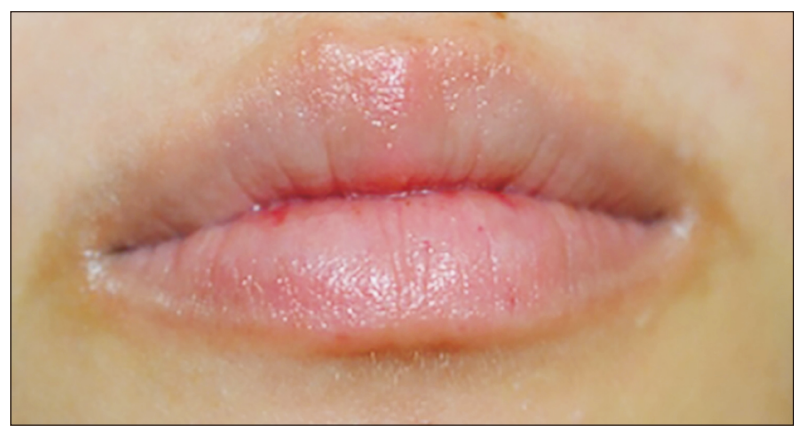

Fig. 2. After 9 laser treatments, the extent and degree of perioral hyperpigmentation observed around the vermilion border were significantly reduced. 
of Ota, nevus of Ito, and black/dark blue type tattoos. ${ }^{5,6}$ In these cases, we usually use high fluence and the endpoint of this treatment is immediate whitening. However, in the case of melasma and post-inflammatory hyperpigmentation, if you use high fluence, the melasma gets worse, so you need to use low fluence. ${ }^{7,8}$

It cleaves melanin granules without cellular damage, diffuses them into the cytoplasm, and induces microdamage of dermis, causing dermal remodeling and proliferation of new collagen, which results in clinical improvement of pigmented lesions and skin rejuvenation. This technique is called laser toning. ${ }^{9}$ To induce this effect to treat perioral hyperpigmentation, we used 1,064-nm Qswitched Nd:YAG laser in low fluence and multipass technique. In addition to the 1,064-nm Q-switched Nd:YAG laser, there is a case of successfully treating idiopathic isolated perioral hyperpigmentation with an erbium:YAG laser. ${ }^{1}$

We report a case of safe and effective treatment of perioral pigmentation by 1,064-nm Q-switched Nd:YAG laser toning.

\section{CONFLICT OF INTEREST}

No potential conflict of interest relevant to this article was reported.

\section{FUNDING}

None.

\section{REFERENCES}

1. Copcu E, Tugay C, Tugay G. Idiopathic isolated perioral hyperpigmentation and treatment with the erbium:YAG laser. Lasers Med Sci 2004;18:223-6.

2. Lenane P, Powell FC. Oral pigmentation. J Eur Acad Dermatol Venereol 2000;14:448-65.

3. Vashi NA, Kundu RV. Facial hyperpigmentation: causes and treatment. Br J Dermatol 2013;169 Suppl 3:41-56.

4. Grunebaum LD, Smith JE, Hoosien GE. Lip and perioral trauma. Facial Plast Surg 2010;26:433-44.

5. Orringer JS. Nonablative laser and light-based therapy: cosmetic and medical indications. In: Kang S, Amagai M, Bruckner AL, Enk AH, Margolis DJ, McMichael AJ, et al, editors. Fitzpatrick's dermatology. 9th ed. New York: McGraw-Hill; 2019. p.3846-56.

6. James WD, Elston DM, Treat J, Rosenbach MA, Neuhaus IM. Andrews' diseases of the skin: clinical dermatology. 13th ed. Edinburgh: Elsevier; 2019. p.909-21.

7. Arora P, Sarkar R, Garg VK, Arya L. Lasers for treatment of melasma and post-inflammatory hyperpigmentation. J Cutan Aesthet Surg 2012;5:93-103.

8. Sim JH, Park YL, Lee JS, Lee SY, Choi WB, Kim HJ, et al. Treatment of melasma by low-fluence $1064 \mathrm{~nm} \mathrm{Q}$-switched Nd:YAG laser. J Dermatolog Treat 2014;25:212-7.

9. Wong Y, Lee SS, Goh CL. Hypopigmentation induced by frequent low-fluence, large-spot-size QS Nd:YAG laser treatments. Ann Dermatol 2015;27:751-5.

How to cite this article: Kim WJ, Cho HK, Lee SJ. Perioral hyperpigmentation treated with 1,064-nm Q-switched neodymium:yttrium-aluminum-garnet laser toning. Med Lasers 2021;10:49-51. https://doi.org/10.25289/ML.2021.10.1.49 